\title{
Lobar torsion after thoracoscopic upper bilobectomy: report of a case
}

\author{
Dario Amore $^{1}$, Antonio Molino ${ }^{2}$, Dino Casazza ${ }^{1}$, Umberto Caterino ${ }^{3}$, Marcellino Cicalese ${ }^{1}$, Roberto \\ Scaramuzzi $^{1}$, Davide Di Natale ${ }^{1}$, Albina Palma ${ }^{4}$, Pasquale Imitazione ${ }^{2}$, Carlo Curcio ${ }^{1}$ \\ ${ }^{1}$ Thoracic Surgery Unit, Monaldi Hospital, Naples, Italy; ${ }^{2}$ Department of Respiratory Diseases, Division of Pneumology, University of Naples \\ Federico II, Monaldi Hospital, Naples, Italy; ${ }^{3}$ Thoracic Endoscopic Unit, Monaldi Hospital, Naples, Italy; ${ }^{4}$ Emergency Department, C.T.O., \\ Naples, Italy \\ Correspondence to: Dario Amore. Thoracic Surgery Unit, Monaldi Hospital, Leonardo Bianchi Street, 1, 80131 Naples, Italy. \\ Email: dario.amore@alice.it.
}

\begin{abstract}
Lung or lobar torsion is a very rare condition that can cause serious complications if missed or untreated. We report a case of a 72-year-old man undergoing video-assisted thoracoscopic upper bilobectomy for treatment of lung cancer. On the first postoperative day radiographic and bronchoscopic findings suggested lobar torsion and then exploratory thoracotomy was carried out within 24 hours of the initial procedure performing simple detorsion and not completion pneumonectomy for various reasons: absence of pulmonary necrosis, incomplete rotation of the bronchovascular pedicle, patient's inability to functionally tolerate a pneumonectomy. Unfortunately the patient didn't show clinical and radiological improvement, during the postoperative period, and he died 12 days later due to cardiorespiratory complications. This case report highlights the need for prompt intervention in patients with lobar torsion within a few hours from the onset of the phenomenon. At the same time it highlights the possibility of fatal outcome in patients unable to functionally tolerate further lung resection. To the best of our knowledge, this is the first report of lobar torsion following video-assisted thoracoscopic upper bilobectomy.
\end{abstract}

Keywords: Lobar torsion; upper bilobectomy; video-assisted thoracoscopic surgery

Received: 28 September 2018; Accepted: 12 October 2018; Published: 12 November 2018.

doi: 10.21037 /jovs.2018.10.17

View this article at: http://dx.doi.org/10.21037/jovs.2018.10.17

\section{Introduction}

Pulmonary torsion is a rare and life-threatening condition in which the twisting of a lung lobe or an entire lung on its bronchovascular pedicle can lead to pulmonary infarction with secondary gangrene if left untreated, due to obstruction of the bronchus and blood vessels $(1,2)$. This rare event, seen mainly following pulmonary resection or other thoracic procedures with an incidence of $0.089-0.2 \%$, can also occur spontaneously, with severe blunt chest trauma or in association with other conditions as diaphragmatic hernia and complicating pneumonia $(3,4)$. A sudden and almost total opacification of a hemithorax on a chest X-ray or an early progressive lobar opacity without signs of atelectasis can raise a suspicion of pulmonary torsion: in this case bronchoscopy and computed tomography (CT) are the most appropriate diagnostic tools (5). Early diagnosis and urgent management can reduce the likelihood of a fatal outcome (6).

\section{Case presentation}

A 72-year-old man with a diagnosis of pulmonary adenocarcinoma, obtained by computed tomographyguided biopsy, was admitted to our unit. Chest CT revealed a right lung lesion crossing the minor fissure and measuring approximately $3.5 \mathrm{~cm} \times 2.5 \mathrm{~cm}$. Positronemission tomography (PET) demonstrated intense focal fluorodeoxyglucose uptake in a right pulmonary mass, without distant metastasis; the standardized uptake value was 6.7. Pulmonary function tests and cardiopulmonary 


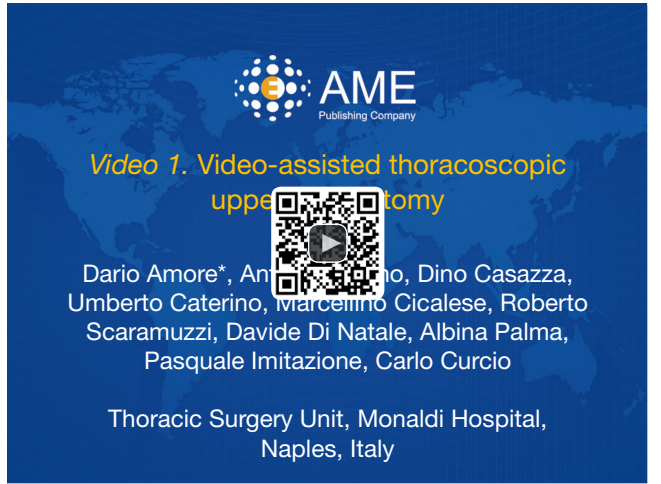

Figure 1 Video-assisted thoracoscopic upper bilobectomy. Preoperative computed tomography scan revealed a right lung lesion crossing the minor fissure in patient with anomalous pulmonary vein coming out from the right upper lobe and draining into the superior vena cava. The video shows the individual dissection of the bronchovascular structures after transection of the anomalous vein using vascular stapler (7).

Available online: http://www.asvide.com/article/view/28213

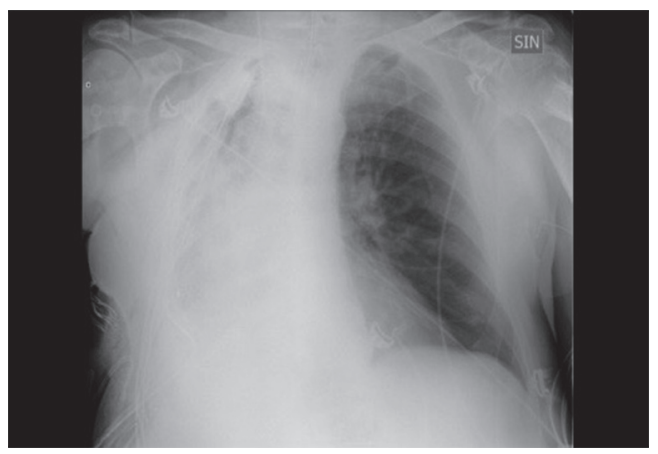

Figure 2 Chest X-ray on the first postoperative day showing complete opacification of the right chest.

exercise test revealed a forced expiratory volume at first second (FEV1) of $1.78 \mathrm{~L}$ (70\% predicted), carbon monoxide lung diffusion capacity (DLCO) of $22.1 \mathrm{~mL} / \mathrm{min} / \mathrm{mmHg}$ (103\% predicted) and maximum oxygen consumption $\left(\mathrm{VO}_{2} \max \right)$ of $10.4 \mathrm{~mL} / \mathrm{Kg} / \mathrm{min}(52 \%$ predicted). The patient, preoperatively staged as cT2aN0M0, underwent video-assisted thoracoscopic upper bilobectomy and systematic lymphadenectomy performed through a three-port anterior approach (Figure 1). The immediate postoperative course was uneventful. The next morning the patient developed tachypnea and, on auscultation, breath sounds were diminished when compared with the left hemithorax. Chest X-ray demonstrated complete

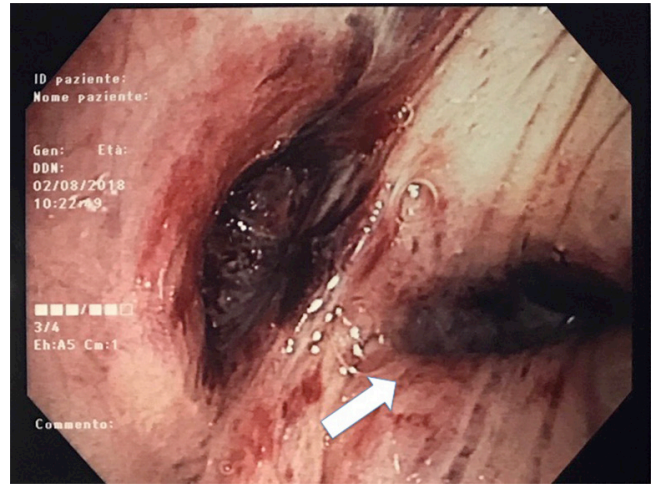

Figure 3 Postoperative flexible bronchoscopy: the orifice of the right lower lobe is distorted and narrowed (white arrow).

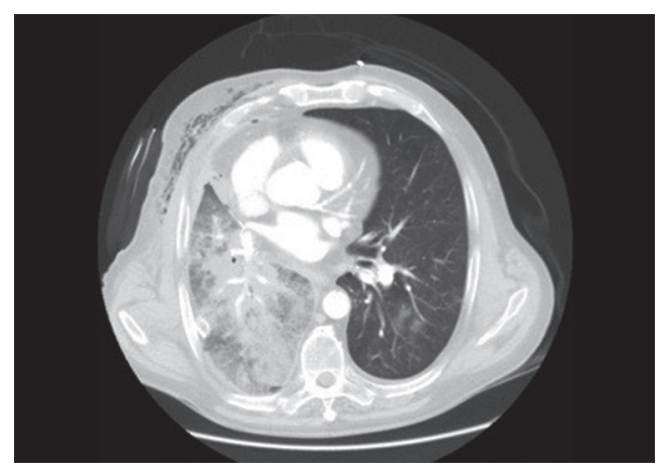

Figure 4 Chest CT demonstrates lobar consolidation, tapered inferior pulmonary vein and acute angulation of the right lung hilum.

opacification of the right hemithorax. (Figure 2). Urgent flexible bronchoscopy revealed that the right lower lobe bronchial orifice was rotated and narrowed (Figure 3). CT of the chest showed right lobar consolidation with ground glass attenuation and obstruction of the right lower lobe bronchus (Figure 4). After ruling out the presence of pulmonary vein thrombosis by a trans-esophageal echocardiography (TEE), immediate exploratory thoracotomy was performed. The right lower lobe was shifted from its original position to the superior side and was rotated less than $180^{\circ}$ degrees clockwise around the bronchovascular pedicle. The lobe was congested with no evidence of pulmonary necrosis. We performed simple detorsion and intraoperative bronchoscopy, after airway suctioning, showed a patent right lower lobe bronchus (Figure 5). As the inferior pulmonary vein pulsed and pulmonary congestion was partially improved, 


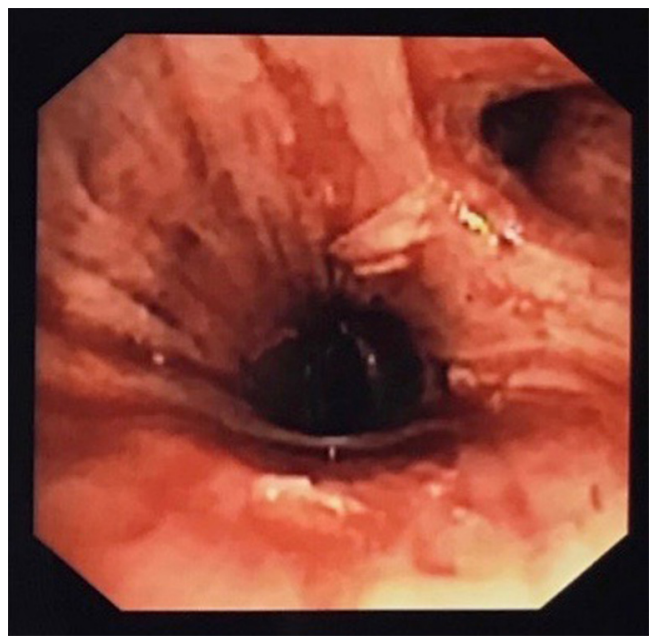

Figure 5 Intraoperative bronchoscopy following detorsion and airway suctioning: right lower lobe bronchus appears to be patent.

we decided to preserve the right lower lobe, taking also into account that the patient wouldn't have been able to tolerate completion pneumonectomy due to not optimal preoperative cardiopulmonary exercise test. Despite surgical reintervention within 24 hours of the initial procedure, in the repeated chest CT scans persistent right lobar consolidation was observed; the patient showed no clinical improvement and died at the intensive care unit 12 days later due to cardiorespiratory complications.

\section{Discussion}

Torsion of a lung or lung lobe on its bronchovascular pedicle is an uncommon but potentially fatal event if left untreated (4). Described predisposing factors in the development of lobar torsion include: transection of the pulmonary ligament, extensive dissection around the hilum, complete interlobar fissure, airless lobe, pneumothorax or pleural effusion, and so on $(2,8)$. The most frequent site of torsion is the middle lobe following right upper lobectomy; however, other lobes can be involved following pulmonary resection. Authors have reported left lower lobe torsion after left upper lobectomy, right lower lobe torsion after right upper lobectomy, left upper lobe torsion after left lower lobectomy and lingulectomy, left common basal pyramid torsion following left upper lobectomy and superior segmentectomy $(4,6,9)$. In addition, thoracic surgeons have reported torsions occurred spontaneously, with chest trauma, or after other thoracic procedures as esophagectomy, hiatal hernia repair and parietal pleurectomy (3). Early detection of lobar torsion is vital for prompt management (10). This rare condition usually presents with non-specific clinical signs but the bedside chest X-ray can provide initial evidence of torsion showing sudden lobar opacification or early progressive lobar opacity without signs of atelectasis. CT findings are more specific including lobar consolidation with ground glass attenuation, acute angulation of the lung hilum with proximal bronchial tapering and enlargement of the pulmonary veins. Direct visualization through bronchoscopy can aid in diagnosis revealing a swollen and distorted bronchial orifice (11). Treatment of lobar torsion includes simple detorsion or surgical resection. Several authors recommend to perform lobectomy of the twisted lung when ischemic necrosis or gangrene occur and when rotation or angulation of the pedicle causes acute obstruction of the bronchus, pulmonary artery and vein $(5,12)$. In our case right lower lobe was repositioned to its natural orientation within 24 hours of the initial procedure. We preserved the lobe for the following reasons: no evidence of pulmonary necrosis; pulmonary congestion partially improved intra-operatively; high postoperative risk after pneumonectomy in patient with a $\mathrm{VO}_{2}$ max\% (percentage of the predicted value of the maximum oxygen consumption) $=52 \%$. Despite reexploration within 24 hours of the initial surgical resection, the patient didn't improve and died due to postoperative cardiorespiratory complications. This case report shows that detorsion, even in the absence of necrosis, should be performed within a few hours following the onset of torsion and, according to some authors, we believe that if this phenomenon persists for more than a few hours, preservation of the involved pulmonary area may not lead to clinical improvement (2). Although many authors argue that surgical resection remains the best treatment in case of delayed intervention $(1,5)$, this option isn't suitable in patients with not optimal cardiopulmonary exercise test for completion pneumonectomy.

\section{Conclusions}

Lung lobe torsion is a rare condition that requires urgent management to avoid catastrophic consequences. Some authors have suggested simple detorsion especially in absence of pulmonary gangrene or complete rotation of the bronchovascular pedicle but the treatment should be performed promptly within a few hours after onset of the phenomenon. Surgical resection, in selected cases, may be 
considered a valid alternative to detorsion but if a patient can't tolerate further lung resection, twisting of the lung lobe can lead to a fatal outcome. Lobar torsion following video-assisted thoracoscopic upper bilobectomy has never been reported to our knowledge.

\section{Acknowledgments}

Funding: None.

\section{Footnote}

Conflicts of Interest: All authors have completed the ICMJE uniform disclosure form (available at http://dx.doi. org/10.21037/jovs.2018.10.17). The authors have no conflicts of interest to declare.

Ethical Statement: The authors are accountable for all aspects of the work in ensuring that questions related to the accuracy or integrity of any part of the work are appropriately investigated and resolved. All procedures performed in studies involving human participants were in accordance with the Helsinki Declaration (as revised in 2013). Written informed consent was obtained from the patient for publication of this case report and any accompanying images.

Open Access Statement: This is an Open Access article distributed in accordance with the Creative Commons Attribution-NonCommercial-NoDerivs 4.0 International License (CC BY-NC-ND 4.0), which permits the noncommercial replication and distribution of the article with the strict proviso that no changes or edits are made and the original work is properly cited (including links to both the formal publication through the relevant DOI and the license). See: https://creativecommons.org/licenses/by-nc-nd/4.0/.

\section{References}

1. Mansour W, Moussaly E, Abou Yassine A, et al. Left

doi: 10.21037 /jovs.2018.10.17

Cite this article as: Amore D, Molino A, Casazza D, Caterino U, Cicalese M, Scaramuzzi R, Di Natale D, Palma A, Imitazione P, Curcio C. Lobar torsion after thoracoscopic upper bilobectomy: report of a case. J Vis Surg 2018;4:236.
Lung Torsion: Complication of Lobar Resection for an Early Stage Lung Adenocarcinoma. Case Rep Crit Care 2016;2016:9240636.

2. Kanemitsu S, Tanaka K, Suzuki H, et al. Pulmonary torsion following right upper lobectomy. Ann Thorac Cardiovasc Surg 2006;12:417-9.

3. Schamaun M. Postoperative pulmonary torsion: report of a case and survey of the literature including spontaneous and posttraumatic torsion. Thorac Cardiovasc Surg 1994;42:116-21.

4. Wong PS, Goldstraw P. Pulmonary torsion: a questionnaire survey and a survey of the literature. Ann Thorac Surg 1992;54:286-8.

5. Apostolakis E, Koletsis EN, Panagopoulos N, et al. Fatal stroke after completion pneumonectomy for torsion of left upper lobe following left lower lobectomy. J Cardiothorac Surg 2006;12;1:25.

6. Wang WL, Cheng YP, Cheng CY, et al. Left common basal pyramid torsion following left upper lobectomy/ segmentectomy. Asian Cardiovasc Thorac Ann 2015;23:474-7.

7. Amore D, Molino A, Casazza D, et al. Video-assisted thoracoscopic upper bilobectomy. Asvide 2018;5:851. Available online: http://www.asvide.com/article/view/28213

8. Alassar A, Marchbank A. Left lower lobe torsion following upper lobectomy-prompt recognition and treatment improve survival. J Surg Case Rep 2014;2014.

9. Chen C, Zheng H, Duan L, et al. Complete lobar torsion simulating hemorrhagic shock after left upper lobectomy. Asian Cardiovasc Thorac Ann 2009;17:191-3.

10. Kelly MV 2nd, Kyger ER, Miller WC. Postoperative lobar torsion and gangrene. Thorax 1977;32:501-4.

11. Demir A, Akin H, Olcmen A, et al. Lobar torsion after pulmonary resection; report of two cases. Ann Thorac Cardiovasc Surg 2006;12:63-5.

12. Lin MW, Huang SC, Kuo SW, et al. Lobar torsion after lung transplantation. J Formos Med Assoc 2013;112:105-8. 